\title{
In silico Recombinant Vaccine Candidate against Coronavirus (2019-nCoV)
}

\author{
Mostafa Norizadehtazehkand ${ }^{1 *}$ and Orkideh Hajipour ${ }^{2}$ \\ ${ }^{1}$ Department of Pharmaceutical Biotechnology, Faculty of Pharmacy, Zonguldak \\ Bulent Ecevit University, Zonguldak, Turkey \\ ${ }^{2}$ Department of Biology, Institute of Basic and Applied Sciences, Pamukkale \\ University, Denizli, Turkey \\ *Corresponding Author: Mostafa Norizadehtazehkand, Department of \\ Pharmaceutical Biotechnology, Faculty of Pharmacy, Zonguldak Bulent \\ Ecevit University, Zonguldak, Turkey.
}

Received: February 06, 2020

Published: March 04, 2020

(C) All rights are reserved by Mostafa

Norizadehtazehkand and Orkideh

Hajipour.

\section{Abstract}

Coronavirus (Wuhan Coronavirus) is a type of virus which has RNA and determined by club like spikes of its surface. The virus is a pathogen virus and it can be infected humans and other animals. This study was aimed to design and analysis of multi epitope vaccine against Wuhan Coronavirus by different bioinformatics analysis and software's.

The vaxiJen score of these sequences were $0.4052,0.4201$, and 0.4371 . The result of Allg Pred and Toxinpred showed that the vaccine does not have allergenic or toxic effect for human cells. The result obtained from Protparam showed that the molecular weight of vaccine is $48.68 \mathrm{kDa}$. The estimated half-life of multi epitope based vaccine was found to be greater than 20 hours in mammalian cells, greater than 30 hours in yeast cells, and greater than 10 hours in E.coli and the candidate vaccine is stable and soluble in water. The membrane helices value of vaccine was $17.73 \%$. Ramachandran plot analysis supported the high quality structure of the refined model. The result of protein protein docking showed the maximum affinity of candidate vaccine to HLA-A0201, HLA - B2705, HLAB5704, and HLA - B57037 with the score of -686.54,-709.05, - 633.53, and 642.61 respectively. The result of this research showed that our designed vaccine maybe stimulate B-Cells and different types of T-cells. However, the vaccine could be produced (in lab scale) and tested in laboratory animals.

Keywords: B-Cell; MHC Class I; Multi Epitope; Recombinant Vaccine; Wuhan Coronavirus

\section{Introduction}

Coronavirus (Wuhan Coronavirus) is a type of virus which has RNA and determined by club like spikes of its surface. The virus is a pathogen virus and it can be infected humans and other animals [1]. The CDC (Centers for Disease Control and Prevention) is tightly monitoring the epidemic disease provoked by a novel coronavirus that was discovered in Wuhan city of China, currently affecting Mainland China along with 27 other countries and territories (CDC, 2020).

The interactions of humans with animals reveal same types of zoonotic infections [2,3]. The incubation time of the Wuhan Coro- navirus vary from 2 to 14 days [4]. It is ambiguous how extensively, the Wuhan Coronavirus can be spread afore symptoms appear [5]. The important symptoms of the virus including coughing, fever, and breathing [6]. As of February fifth, 2020 around 24,553 person have been determained in China [7]. There are a lot of people that are infected but not detected [8,9]. The first spread of the virus outside China happened in Vietnam and the first death outside China was reported in the Philippines [10].

Now, there is only one complete Wuhan Coronavirus genome in NCBI GenBank (with the accession number of MN908947). Five typical ORFs on the similar coding strand were recognized, com- 
prising of ORF1ab polyprotein with 7096 amino acids, membrane protein with 222 amino acids, envelope protein with 75 amino acidss, spike glycoprotein with 1273 amino acid, and nucleocapsid protein with 419- amino acids.

This study was aimed to design and analysis of multi epitope vaccine against Wuhan Coronavirus by different bioinformatics analysis and software's. Finally, molecular docking analysis was used to identify the binding affinity of designed vaccine to HLAA0201, HLA-B2705, HLA-B5704, and HLA-B57037.

\section{Material and Methods}

\section{Prediction of B-cell and T-cell epitopes}

In this study different part of ORF1ab polyprotein (with 7096 amino acids) was obtained from NCBI (NCBI Reference Sequence: NC_045512.2). Before of prediction of epitopes selected fragments sequences were checked by vaxijen. Allertop, and toxinpred server.

B-cell epitopes were designed by IEDB online. In our study the epitopes higher than 0.35 threshold was selected to B-cell [11]. The epitopes were estimated for their binding affinity with predominant HLA- 1 alleles (P-values $<0.05$ were considered significant). Also the oldest vesion of IEDB (2013) webserver was used for prediction of MHC class I epitopes.

\section{Structure of Wuhan Coronavirus vaccine}

The binding affinity of MHC-I and B cell epitopes were taken into selection of suitable epitopes. The predicted B and MHC-I epitopes were linked together by KK (Lysine-Lysine) amino acid linker.

The antigenicity of candidate vaccine was tested by vaxijen webserver [12]. The allergenicity of vaccine was checked by AlgPred online software $[13,14]$. Toxicity analysis of vaccine was done by Toxinpred. Toxinpred analysis software allows to identify the toxic or nontoxic peptides with different length [15].

\section{Analysis of different property of candidate vaccine}

The tendency of peptide vaccine to be soluble in E.coli as overexpression bacterial system, Yeast as eukaryotic host cells, and human cells was analyzed by Protparam [16]. Additionally, molecular weight of designed vaccine, half-life, aliphatic index, isoelectric pointinstability index, and the stability of multi epitope vaccine was checked by Papcolc and Paratparam [17]. In this research for prediction of secondary structure of vaccine and prediction of potential transmembrane helices we used from Parabi webserver.The 3D structure of Wuhan Coronavirus vaccine was drawn by SWISSMODEL and the structure of vaccine was taken in pdb format. The 3D structure of vaccine was refined by 3D refine webserver. We took five different refined model from the server and refined models were checked for 3D refine score, GDT-HA score, GTD-TS, RMSD score, and Mol Probity, and the best structure was selected to docking analysis [18].

The chosen model was analyzed by Ramachandran plot analysis with Procheck [19]. Finally, the binding affinity of epitopes to different types of MHC class I (HLA-A0201, HLA-B2705, HLA-B5704, and HLA-B57037) evaluated by HEX protein protein docking software. The molecular structure of HLA-A0201, HLA-B2705, HLA-B5704, and HLA-B57037 in pdb format was obtained from PDB database. In this study we used pdb format of human serum albumin (protein structure) as negative control and analyzed the affinity of that protein as ligand to HLA-B57037 as receptor in Hex software [20].

\section{Result and Discussion}

Different part of ORF1ab polyprotein of Wuhan Coronavirus sequence was taken from National Center for Biotechnology Information (NCBI). The antigenic property of these sequences was checked by VaxiJen webserver. The antigenic score above the 0.4 were suitable for our study. The vaxiJen score of these sequences were $0.4052,0.4201$, and 0.4371 respectively (Figure 1 ).

The B cell epitopes having a score higher than 0.35 were selected to constriction of recombinant vaccine. The MHC class I epitopes were predicted by IEDB. The epitopes were assessed for their binding affinity with predominant MHC class I alleles. The selected epitopes were analyzed by Vaxijen, AlgPred, and Toxinpred software's. The predicted MHC-I epitopes and B-cell epitopes.

These selected $\mathrm{T}$ cell epitopes have the affinity to different MHC Class I. The amino acid sequence of our designed vaccine with 423 amino acid is shown in figure 2. The result of Allg Pred and Toxinpred showed that the vaccine does not have allergenic or toxic effect for human (Figure 3 and Figure 4). 

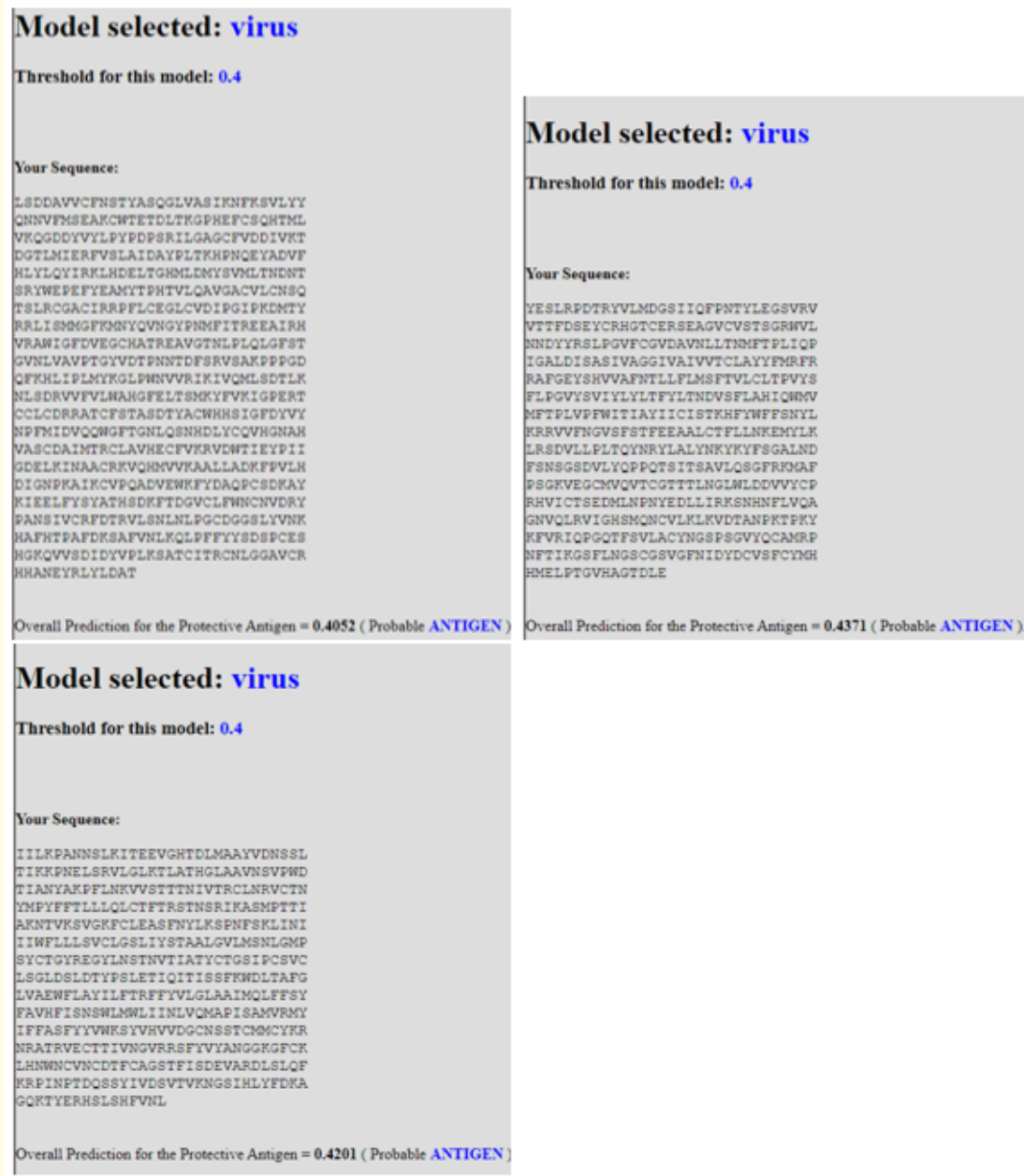

Figure 1: The antigenicity of three fragment of ORF1ab polyprotein.
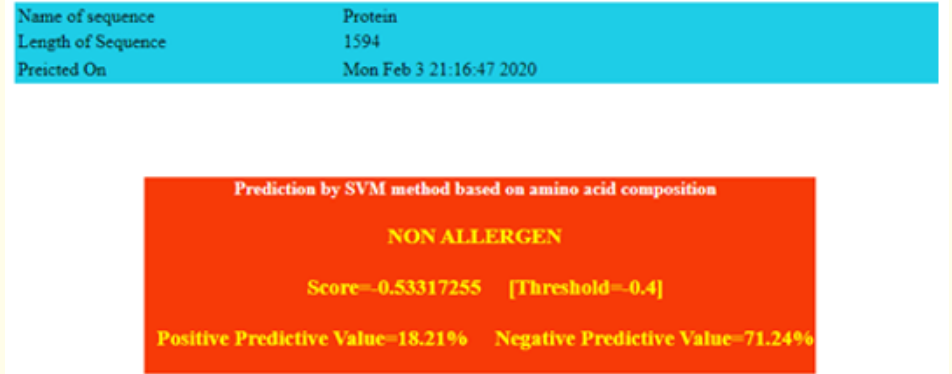

Figure 2: The allergenecity analysis of candidate vaccine. 


\begin{tabular}{|c|c|c|c|c|c|c|c|}
\hline \multicolumn{8}{|c|}{ IVEEAKKVKKKTSAALQPEEEQEEDWLDDDSQQTVGQQDGSEDNQTTTKKDPKLDNYY } \\
\hline \multicolumn{8}{|c|}{ 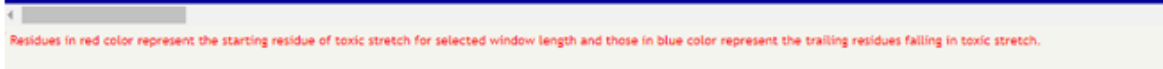 } \\
\hline \multicolumn{8}{|c|}{ Peptides Scanned from Original Protein } \\
\hline Peptide Sequence $\widehat{\imath}$ & SVM score $*$ & Prediction - & Hydrophobicity * & Hydropathicity $\bullet$ & Hydrophilicity $\bullet$ & Charge $*$ & Mol wt $\approx$ \\
\hline IVEEAKKVKK & -0.57 & Non-Toxin & -0.36 & -0.79 & 1.27 & 2.00 & 1171.59 \\
\hline VEEAKKVKKK & -0.82 & Non-Toxin & -0.54 & -1.63 & 1.75 & 3.00 & 1186.60 \\
\hline EEAKKVKKKT & -0.75 & Non-Toxin & -0.61 & -2.12 & 1.86 & 3.00 & 1188.57 \\
\hline EAKKVKKKTS & -0.88 & Non-Toxin & -0.58 & -1.85 & 1.59 & 4.00 & 1146.53 \\
\hline AKKVKKKTSA & -0.82 & Non-Toxin & -0.49 & -1.32 & 1.24 & 5.00 & 1088.49 \\
\hline KKVKKKTSAA & -1.08 & Non-Toxin & -0.49 & -1.32 & 1.24 & 5.00 & 1088.49 \\
\hline KVKKKTSAAL & -1.00 & Non-Toxin & -0.33 & -0.55 & 0.76 & 4.00 & 1073.48 \\
\hline VKKKTSAALQ & -0.86 & Non-Toxin & -0.29 & -0.51 & 0.48 & 3.00 & 1073.44 \\
\hline KKKTSAALQP & -1.03 & Non-Toxin & -0.35 & -1.09 & 0.63 & 3.00 & 1071.42 \\
\hline KKTSAALQPE & -1.06 & Non-Toxin & -0.30 & -1.05 & 0.63 & 1.00 & 1072.36 \\
\hline KTSAALQPEE & .0 .85 & Non-Toxin & .0 .25 & -1.01 & 0.63 & -1.00 & 1073.30 \\
\hline
\end{tabular}

Figure 3: The toxicity analysis of candidate vaccine.

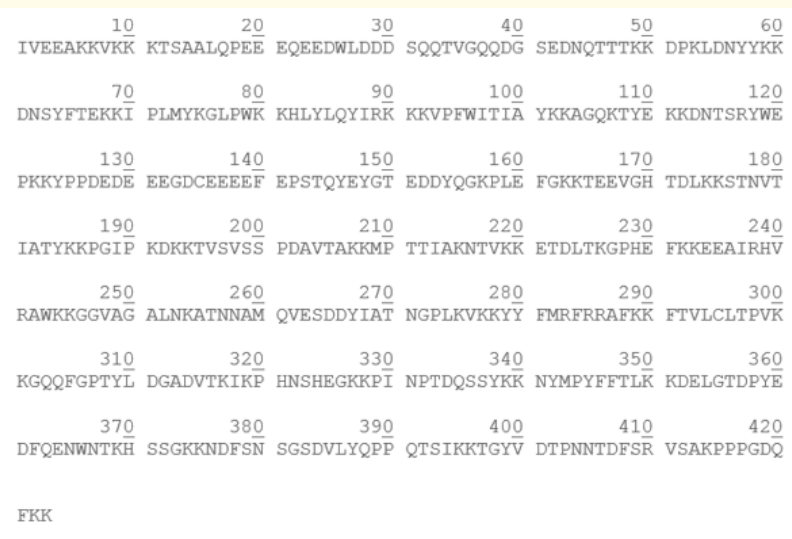

Figure 4: The amino acid sequence of candidate vaccine.

We used from Protparam analysis software for determination of Physicochemical analysis of vaccine. The result obtained from Protparam showed that the molecular weight of vaccine is 48.68 $\mathrm{kDa}$ with 66 total number of negatively charged residues and 77 total number of positively charged residues. The estimated halflife of multi epitope based vaccine was found to be greater than 20 hours in mammalian cells, greater than 30 hours in yeast cells, and greater than 10 hours in E.coli. The vaccine has good instability index because the instability index (II) is computed to 33.03, for this reason the candidate vaccine a stable. The aliphalitic index of our designed vaccine is 46.78 and the grand average of hydropathicity (GRAVY) is -1.237 . So, the designed vaccine is a hydrophilic protein and probably interact with molecules of water. The chemical formula of vaccine is $\mathrm{C}_{2180} \mathrm{H}_{3374} \mathrm{~N}_{574} \mathrm{O}_{677} \mathrm{~S}_{7}$.

The results of Protparam and Pepcalc showed that the vaccine has good water solubility and does not have transmembrane helix. This result revealed that the protein could be cloned and expressed in E.coli or in different host cells. The membrane helices value of vaccine was $17.73 \%$ (Figure 5).

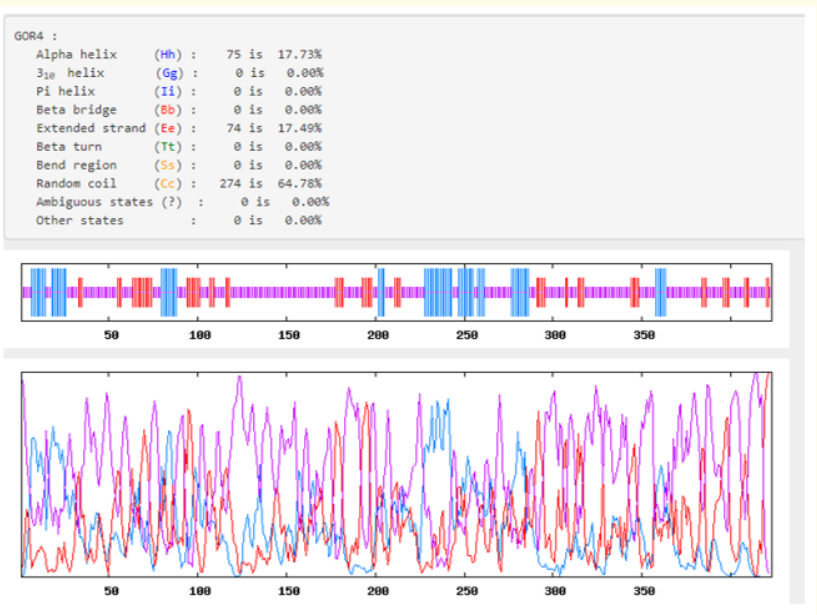

Figure 5: The Secondary structure of candidate vaccine.

The tertiary structure of Coronavirus vaccine was drawn using SWISS-MODEL webserver (Figure 6). The $3^{\text {rd }}$ model was taken 
from SWISS MODEL and refined with 3d Refine software (Figure 7). The best model of $3 \mathrm{~d}$ refine score of vaccine was 5348, GDT-HA score was 1.000, GTD-TS score was 1.000, RMSD score was 0.13, RWPlus score was -9031, and MolProbity score was 1.804 . The refined model of vaccine was examined by Procheck software and the Ramachandran plot was drawn (Figure 8).

The result obtained from Ramachandran analysis of candidate vaccine showed that the $92.6 \%$ of residues are in most favored regions, $7.4 \%$ of residues are additional allowed regions and $0 \%$ of residues is in disallowed regions. The result of Ramachandran plot analysis supported the high quality structure of the refined model.

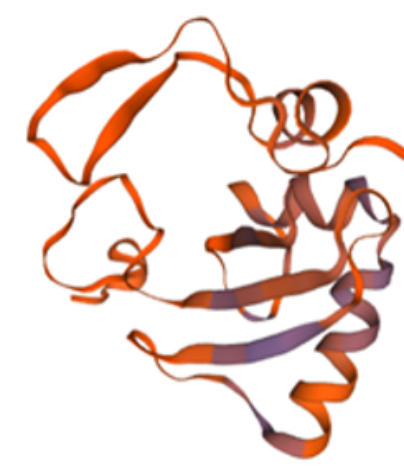

Figure 6: The 3D structure of candidate vaccine.

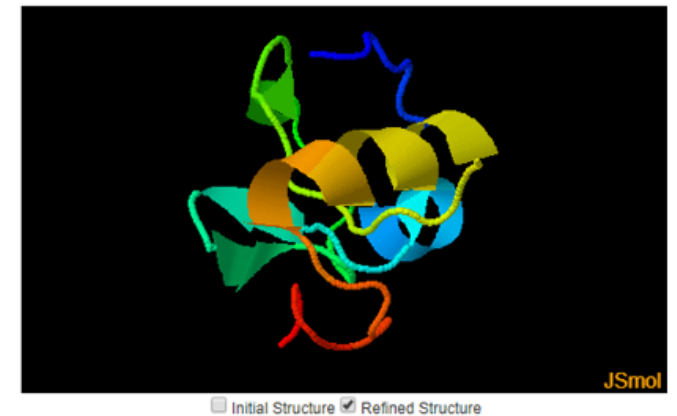

Visualization of refined models are displayed in rainbow colors and initial models in white. Drag the

image to rotate model. Scroll the image to zoom in and out. Right-click to access JSmol functions. Visit the tutorial for more information

\begin{tabular}{ccccccccc} 
Model \# 3 30 $^{\text {refine }}$ Score & GDT-TS & GDT-HA & RMSD (A) & MolProbity & RWPlus - & Refined Model & View Model \\
\hline 4 & 4567.49 & 1.0000 & 1.0000 & 0.242 & 1.635 & -9037.350596 & Refined Model 4 & View \\
\hline 5 & 4517.76 & 1.0000 & 1.0000 & 0.261 & 1.552 & -9032.546531 & Refined Model 5 & View \\
\hline 1 & 5348.88 & 1.0000 & 1.0000 & 0.134 & 1.804 & -9031.612855 & Refined Model 1 & View \\
\hline 2 & 4720.11 & 1.0000 & 1.0000 & 0.187 & 1.635 & -9012.535750 & Refined Model 2 & View \\
\hline 3 & 4625.82 & 1.0000 & 1.0000 & 0.218 & 1.635 & -9007.590339 & Refined Model 3 & View \\
\hline
\end{tabular}

Figure 7: 3D refine models of candidate vaccine.

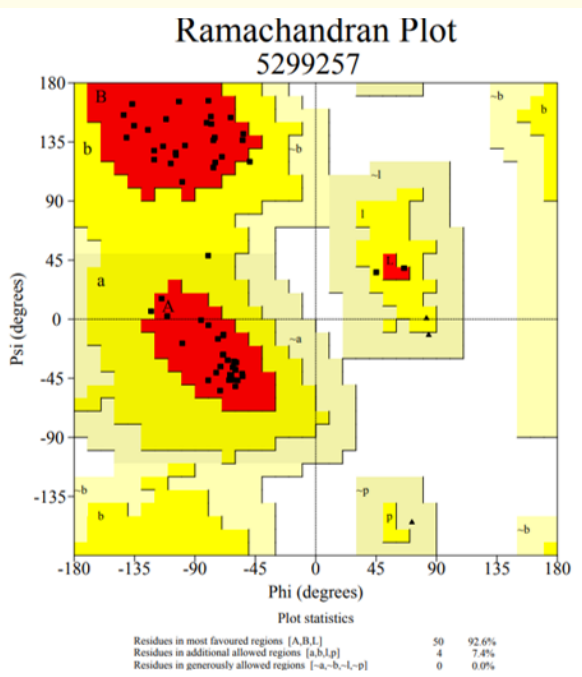

Figure 8: The Ramachandran plot candidate vaccine.
The binding affinity of vaccine to Different HLA of MCH Class I (HLA-A0201, HLA-B2705, HLA-B5704, and HLA-B57037) was investigated by HEX protein docking software. The PDB structure of human serum albumin was used as negative control and the binding affinity of this protein to HLA-B57037 was investigated by the software. The result of protein protein dochong showed that maximum affinity of candidate vaccine to HLA-A0201, HLA-B2705, HLA-B5704, and HLAB57037 with the score of - 686.54, -709.05, - 633.53, and - 642.61 respectively. The affinity of human serum albumin to HLA-B57037 was -94.49. The result of this research showed that our designed vaccine maybe stimulate B-Cells and different types of T-cells (Figure 9 and Figure 10).

In this in silico study immunoinformatical analysis and software's were used to design and checked the vaccine against Wuhan Coronavirus. A physicochemical analysis of vaccine revealed that the vaccine had a molecular weight of $48.6 \mathrm{kDa}$. Dar., et al. reported that the proteins having $<110 \mathrm{kD}$ molecular weight are suitable vaccine [21]. 

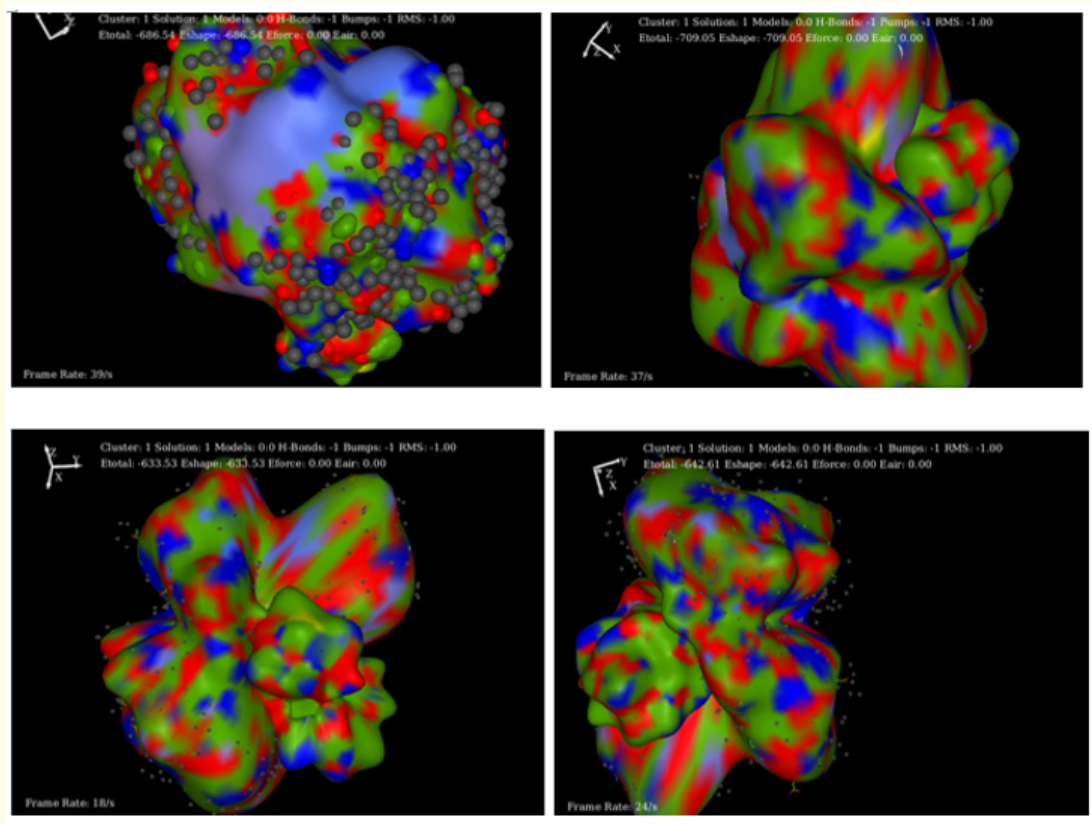

Figure 9: The affinity of candidate vaccine to: A: HLA-A0201 with the energy of -686.54, B: HLA-B2705 with the energy of -709.05,

C: HLA-B5704 with the energy of-633.53, and D: HLA-B57037 with the energy of -642.61.

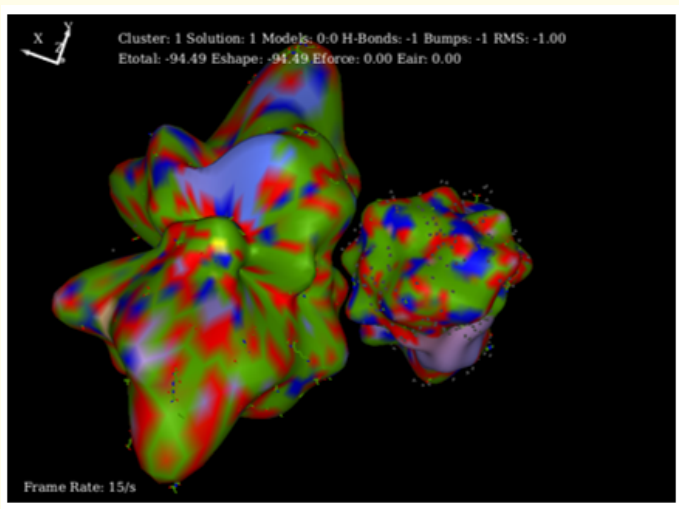

Figure 10: The affinity of bovine serum albumin to -94.49 .

The half-life of designed vaccine was higher than 20 hours in mammalian, greater than 30 hours in yeast cells, and higher than 10 hours in E.coli. The result of this study revealed that the vaccine can be overexpress in E. coli and other host cells. The instability index of our designed vaccine is 33.03 which lower than 40 , so the candidate vaccine is a stable protein. This vaccine does not have toxic and allergenic property, thus the vaccine is not estimated to drop harmful allergic responses in humans. The result of this study showed that the vaccine having the affinity to HLA-A0201, HLAB2705, HLA-B5704, and HLA-B57037.

\section{Conclusion}

The result of our research revealed that the designed vaccine can be suitable against Coronavirus and the vaccine may activate humoral and cellular immune responses. However, the vaccine could be produced (in lab scale) and tested in laboratory animals.

\section{Bibliography}

1. Fung TS and Liu DX. "Human coronavirus: host-pathogen interaction". Annual Review of Microbiology 73 (2019): 529-557.

2. Luk HKH., et al. "Molecular epidemiology, evolution and phylogeny of SARS coronavirus". Infection, Genetics and Evolution (2019): 21-30.

3. Ramadan N and Shaib H. "Middle East respiratory syndrome coronavirus (MERS-CoV): A review". Germs 9 (2019): 35-42. 
4. Wu J T., et al. "Nowcasting and forecasting the potential domestic and international spread of the 2019-nCoV outbreak originating in Wuhan, China: a modelling study". The Lancet (2020).

5. Zhou P., et al. "A pneumonia outbreak associated with a new coronavirus of probable bat origin". Nature (2020): 1-4.

6. Benvenuto D., et al. "The 2019 new Coronavirus epidemic: evidence for virus evolution". Bio Rxiv (2020).

7. WHO. Novel Coronavirus - China. Archived from the original (2020).

8. Operations Dashboard for ArcGIS (2020).

9. Chan J FW., et al. "A familial cluster of pneumonia associated with the 2019 novel coronavirus indicating person-to-person transmission: a study of a family cluster". The Lancet (2020).

10. Rothe C. "Transmission of 2019-nCoV Infection from an Asymptomatic Contact in Germany". NEJM: Correspondence to the Editor Page (2020).

11. Srivastava S., et al. "Structural basis of development of multiepitope vaccine against Middle East respiratory syndrome using in silico approach". Infection and Drug Resistance 11 (2018): 2377-2391.

12. Doytchinova IA and Flower DR. "VaxiJen: a server for prediction of protective antigens, tumor antigens and subunit vaccines". BMC Bioinformatics 8 (2007): 4.

13. Golshani M., et al. "In silico analysis of Shiga toxins (Stxs) to identify new potential vaccine targets for Shiga toxin-producing Escherichia coli". In Silico Pharmacology 5.1 (2016): 2.

14. Adhikari UK., et al. "Immunoinformatics Approach for Epitope-Based Peptide Vaccine Design and Active Site Prediction against Polyprotein of Emerging Oropouche Virus". Journal of Immunology Research (2018): 6718083.

15. Dhanda SK., et al. "Novel in silico tools for designing peptidebased subunit vaccines and immunotherapeutics". Brief Bioin form 18.3 (2017): 467-478.

16. Shey RA., et al. "In-silico design of a multiepitope vaccine candidate against onchocerciasis and related filarial diseases". Scientific Reports 9 (2019): 4409.
17. Khan A., et al. "Computational identification, characterization and validation of potential antigenic peptide vaccines from hrHPVs E6 proteins using immunoinformatics and computational systems biology approaches". PLoS One 13.5 (2018): e0196484.

18. Norizadehtazehkand $\mathrm{M}$ and Hajipour O. "In silico Design a vaccine Candidate against Corynebacterium diphtheria". International Journal of Molecular and Clinical Microbiology 9.1 (2019): 1082-1089.

19. Shahsavani N., et al. "In silico Homology Modeling and Epitope Prediction of NadA as a Potential Vaccine Candidate in Neisseria meningitides". International Journal of Molecular and Cellular Medicine 7.1 (2018): 53-68.

20. Norizadehtazehkand M and Hajipour 0. "Multi Epitope Vaccine Candidate against Mycobacterium Tuberculosis". Drug Designing and Intellectual Properties International Journal (DDIPIJ) (2019).

21. Dar HA., et al. "Immunoinformatics-Aided Design and Evaluation of a Potential Multi-Epitope Vaccine against Klebsiella Pneumoniae". Vaccines (Basel) 7.3 (2019): 88.

\section{Assets from publication with us}

- Prompt Acknowledgement after receiving the article

- Thorough Double blinded peer review

- Rapid Publication

- Issue of Publication Certificate

- High visibility of your Published work

Website: https://www.actascientific.com/

Submit Article: https://www.actascientific.com/submission.php Email us: editor@actascientific.com

Contact us: +919182824667 